International Mathematical Forum, Vol. 8, 2013, no. 30, 1487 - 1492

HIKARI Ltd, www.m-hikari.com

http://dx.doi.org/10.12988/imf.2013.37141

\title{
On Multiplication Lattice Modules
}

\author{
C. S. Manjarekar \\ Department of Mathematics \\ Shivaji University \\ Kolhapur, India \\ csmanjrekar@yahoo.co.in \\ U. N. Kandale \\ Sharad Institute of Techology \\ Yadrav, Ichalakaranji, India \\ ujwalabiraje@gmail.com
}

Copyright (C) 2013 C. S. Manjarekar and U. N. Kandale. This is an open access article distributed under the Creative Commons Attribution License, which permits unrestricted use, distribution, and reproduction in any medium, provided the original work is properly cited.

\begin{abstract}
In this paper, we find some basic results of Multiplication Lattice Modules.
\end{abstract}

Keywords: Multiplicative lattice, lattice module, maximal element, prime element, primary element

\section{Introduction}

Multiplicative lattices are studied by R P Dilworth [3] and D D Anderson [1]. A multiplicative lattice $\mathrm{L}$ is a complete lattice provided with commutative, associative and join distributive multiplication in which the largest element I acts as a multiplicative identity. An element $a \in L$ is called proper if $a<1$. A proper element $\mathrm{p}$ of $\mathrm{L}$ is said to be prime if $a b \leq p$ implies $a \leq p$ or $b \leq p$. An element $m<1$ is called maximal if $m<x \leq 1$ implies $x=1$. If $a \in L, b \in L, \alpha(a: b)$ is the join of all elements $\mathrm{c}$ in $\mathrm{L}$ such that $c b \leq a$. A proper element $\mathrm{p}$ of $\mathrm{L}$ is said to be primary if $a b \leq p$ implies $a \leq p$ or 
$b^{n} \leq p$ for some positive integer n. If $a \in L$, the radical of a denoted by $\sqrt{a}=\vee\left\{x \in L \mid x^{n} \leqslant a, n \in Z_{+}\right\}$. An element $e \in L$ is called meet principal if $(a \wedge(b: e)) e=a e \wedge b$, for all $a, b \in L$. An element $e \in L$ is called join principal if $(a \vee b e): e=(a: e) \vee b$ for all $a, b \in L$ and an element $e \in L$ is called principal if it is both meet and join principal. An element $a \in L$ is called compact if $a \leqslant \vee b_{\alpha}$ implies $a \leqslant b_{\alpha 1} \vee b_{\alpha 2} \vee \ldots \ldots . . \vee b_{\alpha n}$ for some finite subset $\left\{\alpha_{1}, \alpha_{2}, \ldots \ldots \alpha_{n}\right\}^{\alpha}$

If each element of $\mathrm{L}$ is the join of compact elements then $\mathrm{L}$ said to be compactly generated lattice (CG - Lattice) and if each element of L is the join of principal elements then $\mathrm{L}$ said to be principally generated lattice $(\mathrm{PG}$ Lattice). A multiplicative lattice L called r-lattice if it is modular, principally generated, compactly generated and in which the largest element I is compact.

Let $\mathrm{M}$ be a complete lattice and $\mathrm{L}$ be a multiplicative lattice then $\mathrm{M}$ is called L-module or module over $\mathrm{L}$ if there is a multiplication between elements of $\mathrm{L}$ and $\mathrm{M}$ written as $a B$ where $a \in L$ and $B \in M$ which satisfies the following properties,

1. $(a b) B=a(b B)$

2. $\left.\left.\underset{\alpha}{\vee} a_{\alpha}\right) \underset{\beta}{\vee} B_{\beta}\right)=\underset{\alpha, \beta}{\vee} a_{\alpha} B_{\beta}$

3. $I B=B$

4. $O B=O_{M}$, for all $a, a_{\alpha}, b \in L$ and $B, B_{\beta} \in B$, where I is the supremum of $\mathrm{L}$ and $\mathrm{O}$ is the infimum of L.We denote by $0_{M}$ and $I_{M}$ the least element and the greatest element of M.

Let $\mathrm{M}$ be a L-module. If $N \in M$ and $a \in L$ then $(N: a)=\vee\{X \in$ $M \quad \mid a X \leqslant N\}$. If $a, b \in L$, we write $(a: b)=\vee\{x \in L \mid b x \leqslant a\}$ If $A, B \in M$, then $(A: B)=\vee\{x \in L \mid x B \leqslant A\}$. An L-module $\mathrm{M}$ is called a multiplication L-module if for every element $N \in M$ there exists an element $a \in L$ such that $N=a I_{M}$. An element $\mathrm{N}$ of $\mathrm{M}$ is called meet principal if $(a \wedge(B: N)) N=a N \wedge B$ for all $a \in L$ and for all $B \in M$. An element $\mathrm{N}$ of $\mathrm{M}$ is called join principal if $a \vee(B: N)=(a N \vee B): N$ for all $a \in L$ and for all $B \in M$ and $\mathrm{N}$ is said to be principal if it is both meet principal and join principal.

A proper element $\mathrm{N}$ of $\mathrm{M}$ is said to be prime if $a X \leqslant N$ implies $X \leqslant N$ or $a I_{M} \leqslant N$ that is $a \leqslant\left(N: I_{M}\right)$ for every $a \in L, X \in M$. If $\mathrm{N}$ is prime element of $\mathrm{M}$ then $\left(N: I_{M}\right)$ is prime element of $\mathrm{L}[4]$ (proposition (3.6)). An element $N<I_{M}$ in M is said to be primary if $a X \leqslant N$ implies $X \leqslant N$ or $a^{n} I_{M} \leqslant N$ that is $a^{n} \leqslant\left(N: I_{M}\right)$ for some integer n. If $\left(O_{M}: I_{M}\right)=O$ then $\mathrm{M}$ is called faithful L-Module. For $B \in M, \sqrt{B}=\vee\left\{a \in L \mid a^{n} I_{M} \leqslant B\right\}$ for some positive integer n.If each element of $\mathrm{M}$ is the join of principal (compact) 
elements of $\mathrm{M}$ then $\mathrm{M}$ is called principally generated (compactly generated) lattice. Our multiplicative lattice L will be an r-lattice and lattice module M will be a faithful multiplication PG-Lattice (Principally generated) L-Module.

\section{Prime element, primary elements and radi- cals}

Free multiplication modules are studied by Saeed Rajaee [6]. In his paper, he gave the relation between prime and primary ideals of a ring $R$ and the corresponding prime and primary submodules in a module over $\mathrm{R}$. The next theorem gives the relation between a prime element in a multiplicative lattice and a prime element in a lattice module. Similarly, we obtain the relation for primary element in L and primary element in M.

Theorem 2.1. Let $M$ be a lattice module and $p$ be a prime element of $L$ and $q$ be a primary element of $L$ then $\left(I_{M}: p\right)$ is a prime element of $M$ and $\left(I_{M}: q\right)$ is a primary element of $M$. Morever for element $a$ and $b$ of $L, a I_{M} \leqslant b I_{M}$ implies $a \leqslant b$ if and only if $\left(a I_{M}: I_{M}\right)=a$.

Proof. Let $\mathrm{p}$ be a prime element of $\mathrm{L}$ and $a X \leqslant\left(I_{M}: p\right)=Q$. Suppose, $X \nless$ $\left(I_{M}: p\right)$, let $Y \leqslant a I_{M}$. We show that, $Y \leqslant\left(I_{M}: p\right)$ or equivalently, $p Y \leqslant I_{M}$ which is obvious. Hence, $a I_{M} \leqslant\left(I_{M}: p\right)$ and hence, $\left(I_{M}: p\right)$ is prime element of M. Next, let q be a primary element of $\mathrm{L}$ and $a X \leqslant\left(I_{M}: q\right)$. Suppose, $X \nless\left(I_{M}: q\right)$ and $Y \leqslant a^{n} I_{M}$. We show that, $Y \leqslant\left(I_{M}: q\right)$ or equivalently, $q Y \leqslant I_{M}$ which is obvious. Hence, $a^{n} I_{M} \leqslant\left(I_{M}: q\right)$ and $\left(I_{M}: q\right)$ is primary element of M. Suppose, $a I_{M} \leqslant b I_{M}$ implies $a \leqslant b$, we have $a \leqslant\left(a I_{M}: I_{M}\right)$. Let $Y \in S=\left\{x \in L \mid x I_{M} \leqslant a I_{M}\right\}$. Therefore, $Y I_{M} \leqslant a I_{M}$ and hence, $Y \leqslant a$ [2]. This shows that, $\left(a I_{M}: I_{M}\right)=a$. Conversely, let $\left(a I_{M}: I_{M}\right)=a$. If $a I_{M} \leqslant b I_{M}$ then $\left(a I_{M}: I_{M}\right) \leqslant\left(b I_{M}: I_{M}\right)$ and hence, $a \leqslant b$.

In the next result, we obtain another presentation of radical of an element $\mathrm{A}$ of $\mathrm{M}$.

Theorem 2.2. Let $M$ be a multiplication L-Module and element $A \in M$. Then, $\sqrt{A}=\sqrt{q}$ where $A=q I_{M}$.

Proof. We have, $\sqrt{A}=\vee\left\{a \in L \mid a^{n} I_{M} \leqslant A, \quad n \in z_{+}\right\}$[1]. Let $A=q I_{M}$ for some $q \in L$. Then, $\sqrt{A}=\vee\left\{a \in L \quad \mid a^{n} I_{M} \leqslant q I_{M}, n \in z_{+}\right\}=\vee\{a \in L \mid$ $\left.a^{n} \leqslant q, n \in z_{+}\right\}$. So, $\sqrt{A}=\sqrt{q}, \quad q \in L$.

We prove some elementary properties of radicals. For properties of radicals in a multiplicative lattice one can refer $\mathrm{N} \mathrm{K}$ Thakare and C S Manjarekar [7]. 
Theorem 2.3. Let $M$ be a multiplicative L-Module where for any element $A$ and $B$ of $M$,

1. $\sqrt{\sqrt{A}}=\sqrt{A}$

2. $\sqrt{A} \vee \sqrt{B} \leqslant \sqrt{A \vee B}$

3. If $A \vee B=I_{M}$ then $\sqrt{A \vee B}=I_{L}$

4. $\sqrt{A \vee B}=\sqrt{\sqrt{A} \vee \sqrt{B}}$

5. $\sqrt{A \wedge B}=\sqrt{A} \wedge \sqrt{B}$, if multiplication distributes over meet.

Proof. Let $A=a I_{M}$ and $B=b I_{M}$ for some elements a and $\mathrm{b}$ of $\mathrm{L}$, then by theorem $(2.2) \sqrt{A}=\sqrt{a}, \sqrt{B}=\sqrt{b}$. Now, we have,

1. $\sqrt{\sqrt{A}}=\sqrt{\sqrt{a}}=\sqrt{a}=\sqrt{A}$.

2. We have, $\sqrt{A} \vee \sqrt{B}=\sqrt{a} \vee \sqrt{b} ; \quad A \vee B=(a \vee b) I_{M}, \sqrt{A \vee B}=\sqrt{a \vee b}$, $\sqrt{a} \vee \sqrt{b}=\left[\vee\left\{x \in L \mid x^{n} \leqslant a\right\}\right] \vee\left[\vee\left\{x \in L \mid x^{n} \leqslant b\right\}\right]$. Let $x \in S \cup S^{\prime}$ where $S=\left\{x \in L \mid x^{n} \leqslant a\right\}$ and $S^{\prime}=\left\{x \in L \mid x^{n} \leqslant\right.$ b\}.So $x \in S$ or $x \in S^{\prime}$. Then, $x^{n} \leqslant a$ or $x^{m} \leqslant b, n, m \in Z_{+}, n>$ $m$. Hence, $x^{n} \leqslant a \vee b$ and $x \in\left\{y \mid y^{n} \leqslant a \vee b\right\}=S_{1}$. Therefore, $\sqrt{a} \vee \sqrt{b} \leqslant \sqrt{a \vee b}$. This shows that, $\sqrt{A} \vee \sqrt{B}=\sqrt{a} \vee \sqrt{b} \leqslant \sqrt{a \vee b}=$ $\sqrt{A \vee B}$.

3. Let, $A \vee B=I_{M}$. Then, $a I_{M} \vee b I_{M}=(a \vee b) I_{M}=I_{M}$. So, $a \vee b=I_{L}$. Hence, $\sqrt{a \vee b}=\sqrt{I_{L}}$ that is $\sqrt{A \vee B}=I_{L}$

4. We have, $\sqrt{A \vee B}=\sqrt{a I_{M} \vee b I_{M}}=\sqrt{(a \vee b) I_{M}}=\sqrt{a \vee b}$. We know that, $\sqrt{a \vee b}=\sqrt{\sqrt{a} \vee \sqrt{b}}$. Therefore, $\sqrt{A \vee B}=\sqrt{\sqrt{A} \vee \sqrt{B}}$.

5. We have, $\sqrt{A} \wedge \sqrt{B}=\sqrt{a} \wedge \sqrt{b}, \quad A \wedge B=a I_{M} \wedge b I_{M}=(a \wedge b) I_{M}$ (Since, multiplication distributes over meet). But, $\sqrt{A \wedge B}=\sqrt{a \wedge b}=\sqrt{a} \wedge \sqrt{b}$. Therefore, $\sqrt{A \wedge B}=\sqrt{A} \wedge \sqrt{B}$.

The next theorem gives the relation between primary element of lattice module $\mathrm{M}$ and prime element of multiplicative lattice $\mathrm{L}$.

Theorem 2.4. Let $M$ be a multiplication L-module, where for any element $A$ of $M$, if $Q$ is primary element of $M$ then $\sqrt{Q}$ is prime element of $L$.

Proof. Let $Q=q I_{M}$, for some element q of L. Now, $\left(Q: I_{M}\right)=\left(q I_{M}: I_{M}\right)=q$ is a primary element of L. Hence, $\sqrt{q}$ is a prime element of L. Therefore, by theorem $(2.2), \sqrt{Q}=\sqrt{q}$ is prime element of L. 
The next result gives the characterization for elements to be equal.

Theorem 2.5. Let $N_{1}$ and $N_{2}$ be two elements of multiplication lattice module M. Then $\left(N_{1}: I_{M}\right)=\left(N_{2}: I_{M}\right)$ if and only if $N_{1}=N_{2}$.

Proof. Let, $N_{1}=a I_{M}$ and $N_{2}=b I_{M}$ for some element $a, b$ of L.Let, $\left(N_{1}\right.$ : $\left.I_{M}\right)=\left(N_{2}: I_{M}\right)$. Then $\left(a I_{M}: I_{M}\right)=\left(b I_{M}: I_{M}\right)$.Hence, $\mathrm{a}=\mathrm{b}$ and $a I_{M}=$ $b I_{M}$. Conversely, let $N_{1}=N_{2}$. So, $a I_{M}=b I_{M}$ gives $\left(a I_{M}: I_{M}\right)=\left(b I_{M}: I_{M}\right)$ and therefore, $\left(N_{1}: I_{M}\right)=\left(N_{2}: I_{M}\right)$.

One may ask the question whether the prime element is minimal or not.The answer is given in the next theorem.

Theorem 2.6. Let $N$ be a prime element of multiplication L-Module $M$ and $p$ is the prime element of $L$ and $\left(N: I_{M}\right)>p$, then $N$ is not a minimal prime element of $M$.

Proof. Since, $\mathrm{M}$ is a multiplication L module, $N=q I_{M}$ for some element q of L. We have, $\left(N: I_{M}\right)=\left(q I_{M}: I_{M}\right)=q$ is the prime element of L. Let p be a prime element in L. $p=\left(p I_{M}: I_{M}\right)<\left(N: I_{M}\right)$. Therefore, $p I_{M}$ is a prime element of $\mathrm{M}$ contained in $\mathrm{N}[2]$ and hence, $\mathrm{N}$ is not a minimal prime element of $\mathrm{M}$.

The next result follows immediately.

Theorem 2.7. Let $M$ be a multiplication L-module and $N$ be a minimal prime element of $M$ then there is no prime element $p$ of $L$ where $\left(N: I_{M}\right) \geqslant p$.

The next theorem gives a property of local multiplicative lattice.

Theorem 2.8. Let $(L, m)$ be a local multiplicative lattice and $M$ be a multiplication L-Module such that $I_{M} \neq m I_{M}$ then $m I_{M}$ is a maximal prime element of $M$.

Proof. Since, $\mathrm{m}$ is maximal element of $\mathrm{L}$ then $m I_{M}$ is maximal element of $\mathrm{M}$ and hence, it is a prime element [4]. Let $\mathrm{N}$ be prime element such that $N \geqslant m I_{M}$. Then, $\left(N: I_{M}\right) \geqslant\left(m I_{M}: I_{M}\right)=m$. This implies $N=m I_{M}$ Thus, $m I_{M}$ is a maximal prime element of $\mathrm{M}$.

\section{References}

[1] Anderson D D,PhD Thesis.

[2] Callialp F and Tekir U, Multiplication lattice modules, Iranian Journal of Science and Technology, IJST (2011), A4, 309 - 313. 
[3] Dilworth R P, Abstract commutative ideal theory, Pacific J Math, 12(1962), 481-498.

[4] Johnson J A 1970, a - adic completion of noetherian lattice module, Fund Math, 66, 341-371.

[5] Khouja-AI Eaman A, Maximal element and prime element in lattice modules, Damascus University Journal for Basic Sciences, Vol. 19, No 2, 2003.

[6] Saeed Rajee, Some remarks on free multiplication modules, International Journal of Algebra, Vol. 5, 2011 , no. 14, 655-659.

[7] Thakare N K, Manjarekar C S, Radicals and uniqueness theorems in multiplicative lattices with chain conditions, Studia Scientiarum Mathematicarum Hungarica, 18 (1983), 13-19.

Received: July 11, 2013 